\title{
Postoperative New-Onset Atrial Fibrillation following Noncardiac Operations: Prevalence, Complication, and Long-Term MACE
}

\author{
Ofir Koren $\mathbb{D}^{1},{ }^{1,2}$ Rony Hakim, ${ }^{3}$ Asaf Israeli, ${ }^{2}$ Ehud Rozner, ${ }^{3}$ and Yoav Turgeman ${ }^{1,2}$ \\ ${ }^{1}$ Heart Institute, Emek Medical Center, Afula, Israel \\ ${ }^{2}$ Bruce Rappaport Faculty of Medicine, Technion-Israel Institute of Technology, Haifa, Israel \\ ${ }^{3}$ Anesthesia Department, Emek Medical Center, Afula, Israel \\ Correspondence should be addressed to Ofir Koren; drkorenofir@gmail.com
}

Received 25 June 2020; Revised 5 October 2020; Accepted 6 October 2020; Published 14 October 2020

Academic Editor: Andrea P. Rossi

Copyright (C) 2020 Ofir Koren et al. This is an open access article distributed under the Creative Commons Attribution License, which permits unrestricted use, distribution, and reproduction in any medium, provided the original work is properly cited.

Background. Postoperative new-onset atrial fibrillation (POAF) is a common complication following cardiothoracic surgery, but little is known regarding its occurrence and outcome following noncardiothoracic surgery. This study was intended to examine the incidence of POAF in noncardiothoracic surgeries performed under general anesthesia and its effects on the length of hospitalization stay, short-term and long-term morbidity, and mortality. Methodology. We conducted a retrospective observational descriptive study. The study population consists of patients hospitalized in surgical wards from January 2014 to December 2017. Surgery was defined as noncardiac or thoracic procedure conducted under general anesthesia. Results. A total of 24,125 general anesthesia operations were performed at 7 surgical wards. About two-fifth of the operations (40\%) were operated electively, and the rest underwent emergency surgery. The mean age was $63.78 \pm 11.50$, and more than half (56.9\%) of the participants were female. The prevalence of POAF was 2.69 per 1000 adult patients (95\% CI: 2.11-3.43) and vary significantly among wards. The highest prevalence was observed after hip fixation and laparotomy surgeries (54.9 and 26.7 per 1000 patients, respectively). The median length of hospitalization was significantly higher in POAF patients (21.0 vs. 4.8 days, $p<0.001)$. Patients who developed POAF had significantly higher mortality rates, both inhospital (200 vs. 7.56 deaths per $1000, p=0.001)$ and 1 year (261.5 vs. 33.3 per 1000, $p=0.001$, respectively). There was no significant association between outcome and treatment modalities such as rate or rhythm control and anticoagulant use. Conclusion. New-onset AF following noncardiac surgery is rare, yet poses significant clinical implications, both immediate and long-term. POAF is associated with a longer length of hospitalization and a significantly higher mortality rate, both in short- and long-term.

\section{Introduction}

Postoperative atrial fibrillation (POAF) is one of the most common complications in patients undergoing cardiac surgery. Its incidence is estimated to be about $30-60 \%$ of patients. The occurrence of atrial fibrillation after surgery results in longer hospitalization duration, higher morbidity and mortality, frequent recurrences, and long-term risk of stroke [1-3].

POAF has been extensively studied in the context of cardiac surgery (CS) [4-7], but little is known about its prevalence after noncardiac surgery (NCS). It is estimated that the prevalence is between 5 and $10 \%$ of patients and is dependent on many variables, such as age, cardiac dysfunction, and cardiovascular risk factors [8].

POAF is thought to occur as a result of adrenergic stimulation, systemic inflammation, or activation of the autonomous heart nerves during surgery and up to four days later, for various reasons. These reasons include pain, low blood pressure, infection, and bleeding. POAF appears to be associated with myocardial injury or hypertension even before surgery, possibly from hemodynamically alterations or electrolytic disorders [9].

The therapeutic and preventive approach is unclear. A rate control strategy does not appear to differ from rhythm control therapy as far as mortality is concerned $[10,11]$. 
1.1. The Rationale of the Research. The purpose of this study was to examine the incidence of atrial fibrillation following noncardiothoracic surgeries. We also examined the risk factors for the appearance of POAF, its effect on length of hospitalization, inhospital morbidity and mortality, and abnormal cardiovascular events (MACE) in the short- and long-term follow-up.

\section{Materials and Methods}

This was a retrospective observational descriptive study, which was conducted in the heart institute of Emek Medical Center, Afula, Israel. This facility is a secondary, mediumsized medical center, with larger tertiary hospitals close by. As such, a majority of surgeries performed at this center are emergent. The study population consists of patients hospitalized in seven surgical wards (orthopedics, 2 surgical wards, obstetrics and gynecology, plastic surgery, otolaryngology (ENT, ear, nose, and throat), and urology) from January 1, 2014, to the end of December 2017. Surgery was arbitrarily defined by us as a surgical procedure performed under general anesthesia. Procedures performed with local, regional, or spinal anesthesia were excluded. Patients who met the inclusion criteria were divided based on the appearance of POAF. The development of POAF was observed in the 36-hour close monitoring following surgeries in our facility. We have collected data from the hospital's computer systems ("Orion, Ofek, and Chameleon") in accordance with International Diagnostic Code 10-ICD.

Patients eligible for the study (Table 1, inclusion and exclusion criteria) were divided based on the primary endpoint which was defined as the appearance of new atrial fibrillation diagnosed during hospitalization. Secondary end points were major adverse cardiovascular events (MACE) defined as recurrent atrial fibrillation (AF), cerebrovascular accident (CVA), transient ischemic attack (TIA), and pulmonary embolism (PE) hospitalization due to congestive heart failure $(\mathrm{CHF})$ and death at 30 days and at 1 year.

2.1. Ethics. The study was approved by the Ethics Committee of the hospital in accordance with the Helsinki Convention (no. EMC-0088-18). Informed consent was not required due to the confidentiality of patient data.

2.2. Sample Size. Considerations in calculating the sample size were based on several key points: rough assessment of annually conducted surgeries at our medical center and the incidence of POAF known from prior publication. We estimated an arbitrary minimal incidence rate of $2 \%$ for POAF and at least $15 \%$ group difference. Sample size calculation estimated a total of 4520 participants for 95\% CI and $80 \%$ power. Preliminary data indicated a significantly lower incidence rate of POAF which led us to increase the sample size by elongating the study period.

2.3. Statistics. Categorical variables were presented using frequencies and percentages, and these continuous variables were presented using standard distribution indices (mean, standard deviation, median, etc.). Chi-squared test was used for analysis. Differences in the two group's demographic data were tested by $\chi^{2}$ test or Fisher's exact test appropriate for the categorical data and by student's $t$-test or Mann-Whitney $U$ test in the case of nonparametric data for continuous data. The statistical processing will be carried out using SAS 9.4 software. Significance would be obtained if $p<0.05$.

\section{Results}

A total of 30,223 operations were performed at 7 surgical wards during the study period. 6163 patients were not included in the study due to failure to meet the inclusion criteria. A total of 24,125 patients included in the study were divided to two groups based on the diagnosis of AF (Figure 1).

The prevalence of POAF varies significantly, with an average rate of 2.69 per 1000 adult patients (95\% CI: 2.11-3.43). Higher prevalence was observed after hip fixation and abdominal surgeries conducted in orthopedics and general surgery departments. Patients who developed POAF were older $(63.8 \pm 11.5$ vs. $49.8 \pm 18.6, p<0.0001)$ with higher prevalence of smoking $(23.1 \%$ vs. $9 \%, p<0.0001)$, hypertension $(69.2 \%$ vs. $23.3 \%, p<0.0001)$, and diabetes (36.9\% vs. $14.4 \%, p<0.0001)$. Those patients had significantly higher prevalence of ischemic heart disease $(30.8 \%$ vs. $7.2 \%, p<0.0001)$, chronic heart failure (7.7 vs. 0.6, $p<0.0001)$, chronic renal failure (30.8\% vs. 5.4, $p<0.0001)$, anemia $(18.5 \%$ vs. $9.8, p<0.0001)$, and CVA or TIA $(20.0 \%$ vs. $2.4 \%, p<0.0001)$ as compared to the control group (Table 2). The mean BMI was $28.89 \pm 7.31$, and the mean $\mathrm{CHA}_{2} \mathrm{DS}_{2}$-VASc was $3.78 \pm 1.25$.

After development of POAF, 23\% converted spontaneously, and the rest were approached with medical and electrical cardioversion. Cardioversion using pharmacological agents were the most common initial approach $(42.1 \%)$ as compared to rate control (37.5\%) and electrical cardioversion (20.3\%). Electrical cardioversion was the most efficient treatment in restoring sinus rhythm with $69.2 \%$ success rate. About two-fifth (40\%) of patients with POAF were subjected to inappropriate anticoagulant use at discharge. This was mainly seen in patients who were prescribed with direct oral anticoagulants. Physicians tended to discharge patients with a lower dose of DOAC despite objective indications such as renal failure, low body weight, and advanced age. At 1 year of follow-up, we did not see a higher rate of major bleeding events nor ischemic thromboembolic events.

Among the POAF group, about two-fifths (40\%) of the operation were operated electively, and the rest underwent emergency surgery. POAF development was analyzed according to the type of surgery, whether urgent or elective. There was significantly lower survival time in patients who developed POAF after emergent surgery $(p=0.03)$.

There was no difference in the rates 30 days and 1 year MACE between elective and urgent POAF. Seven patients had MACE at 30 days ( $1 \mathrm{PE}$ and 6 recurrent $\mathrm{AF}$ ). Of these, 
TABLE 1: Inclusion and exclusion criteria.

\begin{tabular}{ll}
\hline Criteria for inclusion & \multicolumn{1}{c}{ Criteria for exclusion } \\
\hline & (1) Age <18 years \\
& (2) Surgery not performed using general anesthesia \\
(1) Inpatients who underwent a surgical procedure during the study period & (3) Cardiac- or thoracic-related surgery \\
& (4) Prior diagnosis of AF \\
& (5) Supraventricular arrhythmia excluded AF \\
\end{tabular}

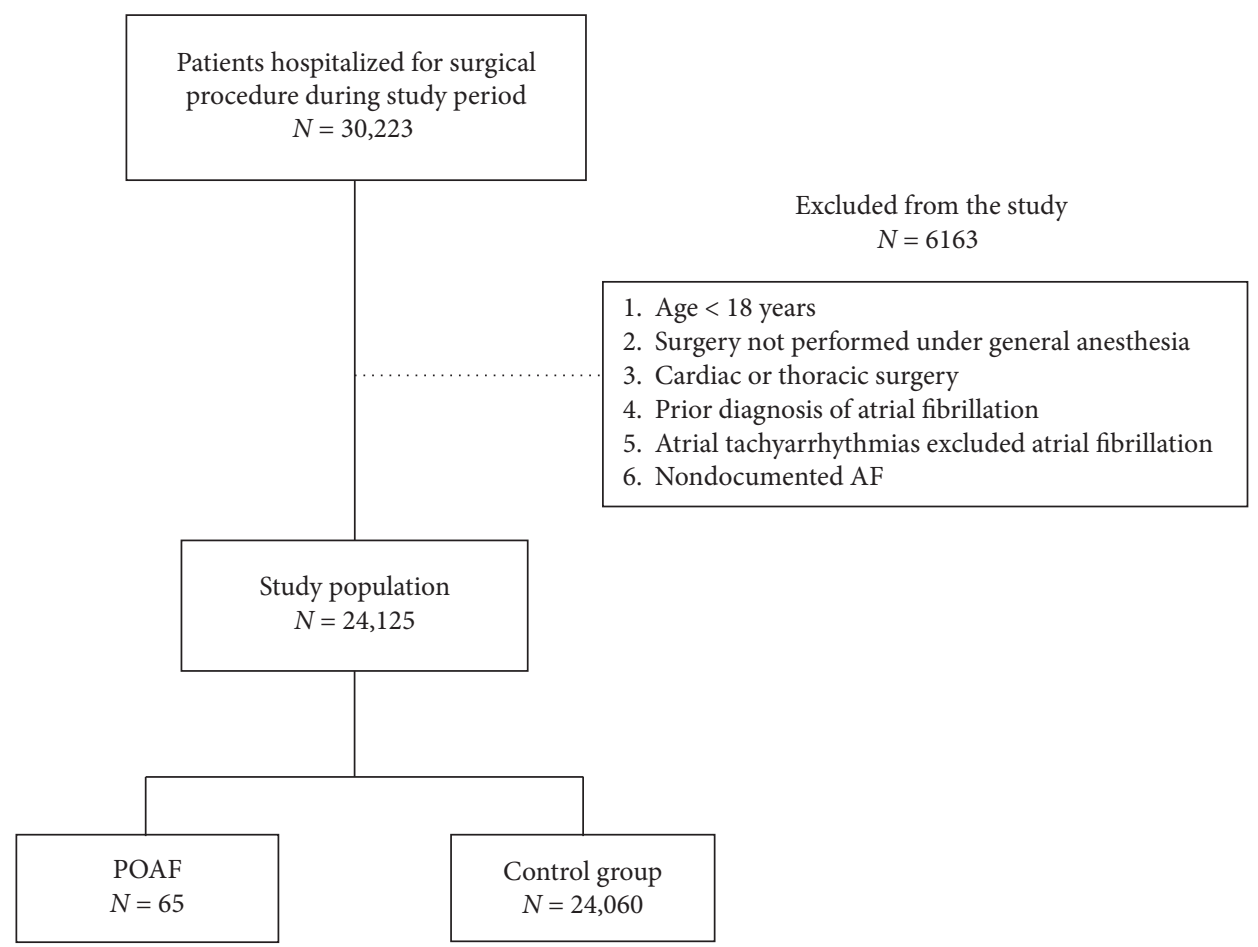

FIGURE 1: Schematic illustration of the study design and patient-selection criteria.

TABLE 2: Baseline clinical characteristics among study groups.

\begin{tabular}{lccc}
\hline Parameters & $\begin{array}{c}\text { POAF } \\
(N=65)\end{array}$ & $\begin{array}{c}\text { Control } \\
(N=24060)\end{array}$ & $p$ value \\
\hline Age (mean \pm range) & $63.8 \pm 11.5$ & $49.8 \pm 18.6$ & $<0.0001$ \\
$(65 ; 40-96)$ & $(49 ; 19-104)$ & \\
Gender: male & $28(43.1)$ & $10877(45.2)$ & 0.73 \\
Smoking & $15(23.1)$ & $2170(9.0)$ & $<0.0001$ \\
Hypertension & $45(69.2)$ & $5602(23.3)$ & $<0.0001$ \\
Diabetes mellitus & $24(36.9)$ & $3454(14.4)$ & $<0.0001$ \\
Renal failure & $20(30.8)$ & $1299(5.4)$ & $<0.0001$ \\
Anemia & $12(18.5)$ & $2357(9.8)$ & $<0.0001$ \\
Ischemic heart & $20(30.8)$ & $1724(7.2)$ & $<0.0001$ \\
disease & $5(7.7)$ & $143(0.6)$ & $<0.0001$ \\
Chronic heart & $13(20.0)$ & $574(2.4)$ & $<0.0001$ \\
failure & $3.78 \pm 1.97$ & $1.24 \pm 1.33$ & $<0.0001$ \\
CVA/TIA & $(3 ; 0-8)$ & $(1 ; 0-9)$ & \\
CHADS $_{2}$-VASC & & & \\
\hline
\end{tabular}

CVA, cerebrovascular accident; TIA, transient ischemic attack. ${ }^{x}$ Defined as serum creatinine level $>1.5 \mathrm{mg} / \mathrm{dL}$ or estimated GFR $<60 \mathrm{ml} / \mathrm{L} / 1.73 \mathrm{~m}^{2}$ using MDRD. ${ }^{\psi}$ Defined as hemoglobin level $<9$ gram $/ \mathrm{dL}$.
MACE occurred in three patients who had elective surgery $(11.5 \%)$ and in four patients who had an emergent surgery $(10.2 \%)$. There was no statistically significant difference in survival time between patients who had elective surgery (28.9 days) and those who had emergent surgery (28.7 days; Mantel-Cox chi-square $=0.02 ; p>0.89)$. This remained true after correcting for age (chi-square $=0.02, p>0.90$ ).

15 patients had 1-year MACE. Of these, MACE occurred in 4 patients who had elective surgery (15.4\%) and in 11 patients who had an emergent surgery $(28.2 \%)$. There was no statistically significant difference in survival time between patients who had elective surgery (312.8 days) and those who had emergent surgery (293.6 days; Mantel-Cox chisquare $=1.23 ; p>0.27)$. This remained true after correcting for age (chi-square $=1.19, p>0.28)$.

There was a significantly higher length of hospitalization in the urgent surgery POAF group $(p=0.008)$. Of the patients who developed POAF, urgent surgery entailed significantly lower survival time (Mantel-Cox chisquare $=4.51, p=0.03$ ). 
Inhospital mortality differed significantly among study groups. 182 patients in the control group died during hospitalization as compared to 13 patients in the POAF group (200 vs. 7.56 per $1000 ; p=0.001$ ). The control group had statistically significant longer survival time than the POAF group for inhospital death (Mantel-Cox chisquare $=14.33 ; p<0.001)$. After correcting for age (Cox regression model), the control group still had a statistically significant longer survival time (chi-square $=7.51 ; p<0.006)$ (Figure 2).

30 days mortality significantly differed among two groups, with 239 patients in the control group died within 30 days and 7 patients in the POAF group (8.22 vs. 107.69 per 1000, $p=0.001$; Mantel-Cox chi-square $=81.26, p<0.001)$. After correcting for age, the difference remained (chisquare $=10.62, p<0.001)$. After correcting for smoking, hypertension, diabetes mellitus, chronic renal failure, anemia, ischemic heart disease, chronic heart failure, CVA/TIA, and $\mathrm{CHADS}_{2}-\mathrm{VA}_{2} \mathrm{SC}$ score, the difference remained (chisquare $=6.51, p<0.01)$.

1 -year mortality differed significantly as well. 802 patients in the control group died at 1 year as compared to 17 patients in the POAF group (261.5 vs. 3.33 per 1000, $p=0.001$ ). Age was not a significant predictor of 1 -year mortality.

Patients who develop POAF had a significantly longer hospital stay. $\mathrm{LOH}$ in the POAF group was 21.0 days (SD: 22.7; range $2-115$ days; $95 \%$ CI: $15.4-26.6$ days) compared to 4.8 days (SD: 7.5 ; range $0-188$ days; $95 \%$ CI: $4.7-4.9$ ) in the control group (Figure 3 ).

In patients developing POAF, 24.6\% developed MACE (1.5\% MI, 1.5\% PE, 12.3\% CHF, $13.5 \% \mathrm{AF}$, and $4.6 \% \mathrm{CVA} /$ TIA).

\section{Discussion}

Postoperative atrial fibrillation in noncardiothoracic surgery is rare, as previously reported [12-20], with a prevalence of 2.69 per 1000 adult patients. The highest prevalence was observed during abdominal and orthopedic surgeries.

The low incidence of POAF may be explained by several factors such as baseline patient characteristics, shorter length of hospitalization, less cardiac trauma and manipulation, and shorter and less accurate cardiac monitoring outside intensive cardiac care.

One must bear in mind, that noncardiothoracic surgeries are usually less major, do not include extensive sternotomies, entail less blood loss, less fluid, and electrolyte imbalance, and most importantly, do not involve extensive cardiac manipulation. Moreover, many noncardiothoracic surgeries include minimally invasive techniques, whereas its usage in cardiothoracic surgeries is still limited. All the aforementioned were found to contribute to the appearance of AF [21-28].

In this study, patients who developed POAF had the same well-known predisposing factors for AF, regardless of surgeries. These patients were older with a significant higher cardiovascular risk profile, with higher prevalence of smoking, hypertension, and diabetes. In addition, these patients were more likely to have prior cardiac injury such as ischemic heart disease infarction, structural changes, and reduced left ventricle function. Therefore, we recommend surgeons to make note of this population and be wary of the potential risk.

The length of hospitalization (LOH) was significantly higher in patients who developed POAF. The mean duration was 21 days, compared to an overall mean duration of less than 5 days. This difference in $\mathrm{LOH}$ among groups could be explained in three possible ways, to the best of our knowledge. One is that AF constitutes a trigger that may lead to further deterioration and longer hospital stay. Second is the hypothesis that AF may be a marker of worse condition and secondary adrenergic surge. Finally, considering that $\mathrm{LOH}$ correlated with increased $\mathrm{CHA}_{2} \mathrm{DS}_{2}$-VASc scores, it is also possible that comorbidities might be the culprit for $\mathrm{LOH}$, and POAF could be ominous of future prognosis.

The incidence of POAF was not found to be unexceptionally common but entailed important prognostic significance. Although rare, it must not be overlooked, and we advise surgeons to obtain cardiologic consult once it develops. Moreover, we encourage that susceptible patients with risk factors, mainly ones undergoing hip fixation or laparotomy, must be carefully examined prior to the operation and after the operation, with special care to this outcome, as it poses short- and long-term ramifications. In addition, patients should be advised to take note if any AFrelated symptoms occur immediately after surgery and to inform their surgeons in such instances.

To the best of our knowledge, there are no evidencebased guidelines concerning this issue, regarding the evaluation of this phenomenon and how to address it. Once POAF has indeed developed, we recommend that, in addition to the routine care of $\mathrm{AF}$, an additional cardiac monitoring should take place, preferably with a Holter monitor for another 48-hour period to examine and study the nature of the AF. Moreover, due to its long-term implications, we advise more frequent cardiac assessments, conducted by either the primary-care physician or a cardiologist, at least in the first year following surgery. Lastly, the risks of antiarrhythmic drugs are well-known [29], especially in elderly patients. We presume that, in light of the scarcity of POAF found in our study, we cannot recommend the use of preoperative prophylactic antiarrhythmic therapy. Nonetheless, if the AF is recurrent during Holter monitoring or later on, we clearly recommend it to be treated as any other AF regarding the use of antiarrhythmics.

\section{Study Limitations}

We used a retrospective methodology using data from computerized systems. Data were obtained with no ability to assess its reliability. Moreover, the follow-up to observe the incidence and development of POAF was made immediately after surgery, during the routine 36-hour postoperative monitoring alone, and therefore, the rate of POAF might be underestimated as the late development of POAF might have been underdiagnosed. 


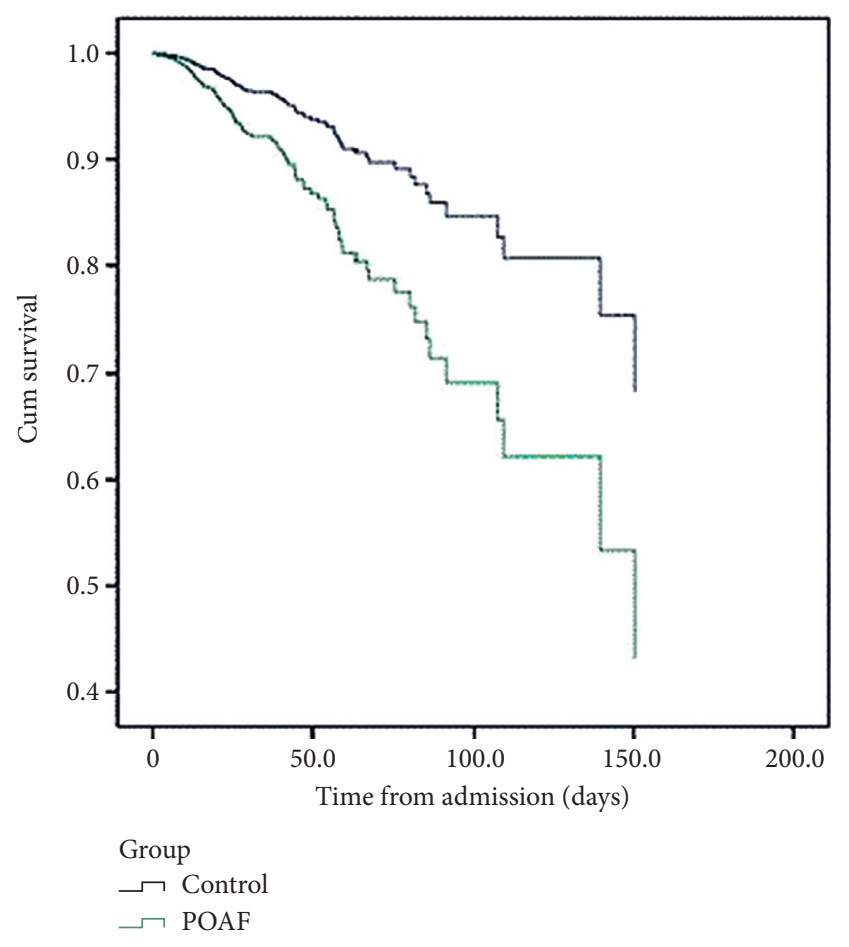

FIgURE 2: Kaplan-Mayer survival curve among study groups.

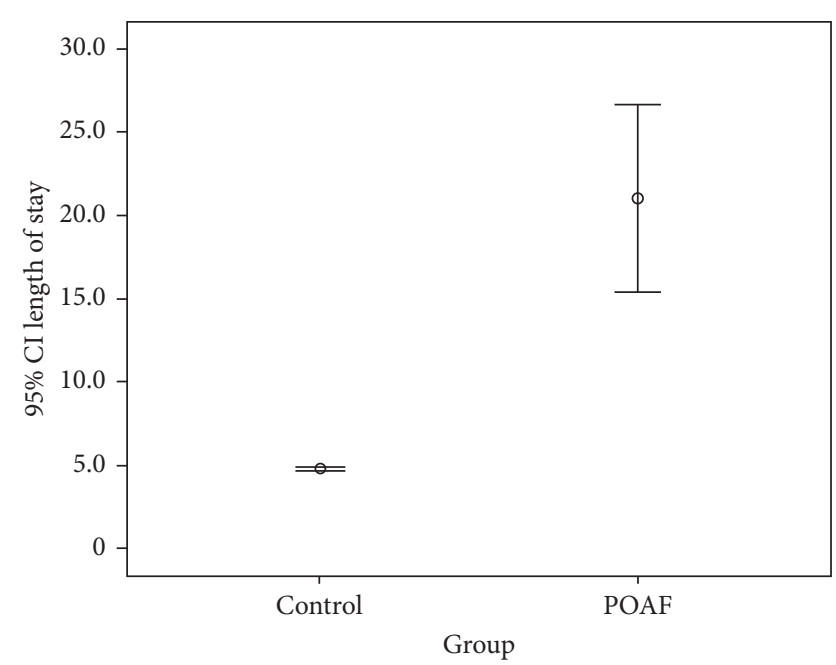

Figure 3: Mean length of stay among study groups.

This study did not examine non-GA operations since we did not have the same rigorous follow-up monitoring as is routinely performed after GA operations, making the information less accurate. In our view, future studies investigating this phenomenon in these operations may be warranted and could possibly provide interesting, additional insights on the subject.

\section{Conclusion}

Our results indicate that POAF following noncardiothoracic surgeries is rare; however, just like postcardiac surgery, it poses a significantly poor prognostic implication. POAF is associated with a fivefold hospitalization length and a significantly higher mortality rate, both inhospital and up to one year following discharge.

\section{Data Availability}

The data used to support the findings of this study are available from the corresponding author upon request.

\section{Conflicts of Interest}

The authors declare no conflicts of interest.

\section{Authors' Contributions}

Ofir Koren and Rony Hakim contributed equally to this work.

\section{References}

[1] D. Antonelli, D. Peres, N. A. Freedberg, A. Feldman, and T. Rosenfeld, "Incidence of postdischarge symptomatic paroxysmal atrial fibrillation in patients who underwent coronary artery bypass graft: long-term follow-up," Pacing and Clinical Electrophysiology, vol. 27, no. 3, pp. 365-367, 2004.

[2] S. Attaran, M. Shaw, L. Bond, M. D. Pullan, and B. M. Fabri, "Atrial fibrillation postcardiac surgery: a common but a morbid complication," Interactive Cardiovascular and Thoracic Surgery, vol. 12, no. 5, pp. 772-777, 2011.

[3] G. Gialdini, K. Nearing, P. D. Bhave et al., "Perioperative atrial fibrillation and the long-term risk of ischemic stroke," JAMA, vol. 312, no. 6, pp. 616-622, 2014.

[4] P. D. Bhave, L. E. Goldman, E. Vittinghoff, J. Maselli, and A. Auerbach, "Incidence, predictors, and outcomes associated with postoperative atrial fibrillation after major noncardiac surgery," American Heart Journal, vol. 164, no. 6, pp. 918-924, 2012.

[5] N. Echahidi, P. Pibarot, G. O'Hara, and P. Mathieu, "Mechanisms, prevention, and treatment of atrial fibrillation after cardiac surgery," Journal of the American College of Cardiology, vol. 51, no. 8, pp. 793-801, 2008.

[6] F. Philip, M. Becker, J. Galla, E. Blackstone, and S. R. Kapadia, "Transient post-operative atrial fibrillation predicts short and long term adverse events following CABG," Cardiovascular Diagnosis and Therapy, vol. 4, no. 5, pp. 365-372, 2014.

[7] G. Tokmaji, R. S. McClure, T. Kaneko, and S. F. Aranki, "Management strategies in cardiac surgery for postoperative atrial fibrillation: contemporary prophylaxis and futuristic anticoagulant possibilities," Cardiology Research and Practice, vol. 2013, Article ID 637482, 16 pages, 2013.

[8] K. Ayoub, F. Habash, A. Almomani et al., "Long term risk of recurrent atrial fibrillation and ischemic stroke after postoperative atrial fibrillation complicating cardiac and noncardiac surgeries," Journal of Atrial Fibrillation, vol. 10, no. 6, p. 1660, 2018.

[9] K. K. Joshi, M. Tiru, T. Chin, M. T. Fox, and M. S. Stefan, "Postoperative atrial fibrillation in patients undergoing noncardiac non-thoracic surgery: a practical approach for the hospitalist," Hospital Practice (1995), vol. 43, no. 4, pp. 235-244, 2015.

[10] T. Omae and Y. Kanmura, "Management of postoperative atrial fibrillation," Journal of Anesthesia, vol. 26, no. 3, pp. 429-437, 2012. 
[11] A. M. Gillinov, E. Bagiella, A. J. Moskowitz et al., "Rate control versus rhythm control for atrial fibrillation after cardiac surgery," The New England Journal of Medicine, vol. 374, no. 20, pp. 1911-1921, 2016.

[12] D. Brathwaite and C. Weissman, "The new onset of atrial arrhythmias following major noncardiothoracic surgery is associated with increased mortality," CHEST Journal, vol. 114, pp. 462-13, 1998.

[13] G. H. Sohn, D. H. Shin, K. M. Byun et al., "The incidence and predictors of postoperative atrial fibrillation after noncardiothoracic surgery," Korean Circulation Journal, vol. 39, pp. 100-104, 2009.

[14] C. A. Polanczyk, L. Goldman, E. R. Marcantonio, E. J. Orav, and T. H. Lee, "Supraventricular arrhythmia in patients having noncardiac surgery: clinical correlates and effect on length of stay," Annals of Internal Medicine, vol. 129, no. 4, pp. 279-285, 1998.

[15] G. S. Batra, J. Molyneux, and N. A. Scott, "Colorectal patients and cardiac arrhythmias detected on the surgical high dependency unit," Annals of the Royal College of Surgeons of England, vol. 83, no. 3, pp. 174-176, 2001.

[16] S. R. Walsh, J. E. Oates, J. A. Anderson et al., "Postoperative arrhythmias in colorectal surgical patients: incidence and clinical correlates," Colorectal Disease, The Official Journal of the Association of Coloproctology of Great Britain and Ireland, vol. 8, no. 3, pp. 212-216, 2006.

[17] K. K. Christians, B. Wu, E. J. Quebbeman, and K. J. Brasel, "Postoperative atrial fibrillation in noncardiothoracic surgical patients," American Journal of Surgery, vol. 182, no. 6, pp. 713-715, 2001.

[18] R. L. Kahn, M. J. Hargett, B. Urquhart et al., "Supraventricular tachyarrhythmias during total joint arthroplasty. Incidence and risk," Clinical Orthopaedics and Related Research, vol. 296, pp. 265-269, 1993.

[19] J. J. Curtis, B. M. Parker, C. A. McKenney et al., "Incidence and predictors of supraventricular dysrhythmias after pulmonary resection," The Annals of Thoracic Surgery, vol. 66, no. 5, pp. 1766-1771, 1998.

[20] S. R. Walsh, T. Tang, C. Wijewardena et al., "Postoperative arrhythmias in general surgical patients," Annals of the Royal College of Surgeons of England, vol. 89, no. 2, pp. 91-95, 2007.

[21] I. M. Danelich, J. M. Lose, S. S. Wright et al., "Practical management of postoperative atrial fibrillation after noncardiac surgery," Journal of the American College of Surgeons, vol. 219, no. 4, pp. 831-841, 2014.

[22] L. Goldman, "Supraventricular tachyarrhythmias in hospitalized adults after surgery. Clinical correlates in patients over 40 years of age after major noncardiac surgery," Chest, vol. 73, pp. 450-454, 1978.

[23] C. Chelazzi, G. Villa, and A. R. De Gaudio, "Postoperative atrial fibrillation," International Scholarly Research Notices Cardiology, vol. 2011, Article ID 203179, 10 pages, 2011.

[24] J. D. Edwards and R. G. Wilkins, "Atrial fibrillation precipitated by acute hypovolaemia," British Medical Journal (Clinical Research Ed), vol. 294, no. 6567, pp. 283-284, 1987.

[25] K. M. Heintz and S. M. Hollenberg, "Perioperative cardiac issues: postoperative arrhythmias," The Surgical Clinics of North America, vol. 85, no. 6, pp. 1103-1114, 2005.

[26] D. R. Van Wagoner, "Electrophysiological remodeling in human atrial fibrillation," Pacing and Clinical Electrophysiology: PACE, vol. 26, no. 7 Pt 2, pp. 1572-1575, 2003.

[27] M. J. Lab, "Contraction-excitation feedback in myocardium. Physiological basis and clinical relevance," Circulation Research, vol. 50, no. 6, pp. 757-766, 1982.
[28] J. R. Ehrlich, S. H. Hohnloser, and S. Nattel, "Role of angiotensin system and effects of its inhibition in atrial fibrillation: clinical and experimental evidence," European Heart Journal, vol. 27, no. 5, pp. 512-518, 2006.

[29] D. M. Roden, "Risks and benefits of antiarrhythmic therapy," New England Journal Of Medicine, vol. 331, no. 12, pp. 785791, 1994. 